\title{
TOPOLOGICAL SCHUR LEMMA AND RELATED RESULTS
}

\author{
BY THEODORE CHANG AND TOR SKJELBRED \\ Communicated by Paul S. Mostert, February 23, 1973
}

We announce here some results of a paper to appear elsewhere [1].

Let a torus $T$ act continuously on a topological space $X$. Let $X \rightarrow X_{T} \rightarrow{ }^{\pi} B_{T}$ be the fibre bundle with fibre $X$ associated (by means of the action of $T$ on $X$ ) to the universal principal $T$ bundle $T \rightarrow E_{T} \rightarrow B_{T}$. We define the equivariant cohomology ring $H_{T}^{*}(X)=H^{*}\left(X_{T}\right)$ where $H^{*}$ denotes Čech cohomology with rational coefficients. When $Y$ is an invariant subspace of $X$, we define $H_{T}^{*}(X, Y)=H^{*}\left(X_{T}, Y_{T}\right)$. Then $R=H^{*}\left(B_{T}\right)$ is a polynomial ring and $H_{T}^{*}(X, Y)$ is a module over $R$ by means of $\pi^{*}$.

For each subtorus $L$ of $T$ let $P L$ be the kernel of $H^{*}\left(B_{T}\right) \rightarrow H^{*}\left(B_{L}\right)$. Let $X^{L}=F(L, X)$ be the set of points fixed by $L$. We will assume that $X$ is compact. Given a closed invariant subspace $Y \subset X$ and an element $x \in H_{T}^{*}(Y)$, we define

$$
\begin{aligned}
& I_{x}=\left\{a \in R \mid a x \text { lies in the image of } H_{T}^{*}(X) \rightarrow H_{T}^{*}(Y)\right\}, \text { and } \\
& I_{x}^{L}=\left\{a \in R \mid a x \text { lies in the image of } H_{T}^{*}\left(X^{L} \cup Y\right) \rightarrow H_{T}^{*}(Y)\right\} .
\end{aligned}
$$

When $L \subset K$ are subtori, $I_{x} \subset I_{x}^{L} \subset I_{x}^{K}$. We say that $K$ belongs to $x$ if $K$ is maximal with respect to the property $I_{x}^{K} \neq R$.

1. THEOREM. The isolated primary components of the ideal $I_{x}$ are the ideals $I_{x}^{K}$ where $K$ belongs to $x$. The radical of $I_{x}^{K}$ is $P K$, hence $\sqrt{I_{x}}=\bigcap P K$ where $K$ ranges over the subtori belonging to $x$.

2. CoRollary. If $I_{x}$ is principal, the subtori belonging to $x$ are all of corank 1 and $I_{x}=\bigcap I_{x}^{K}$ where $K$ ranges over the subtori belonging to $x$. For each such $K, I_{x}^{K}=\left(\omega^{d}\right)$ where $d \geqq 1$ and $\omega \in H^{2}\left(B_{T}\right)$ generates $P K$.

Assume that the fixed point set $F$ of the $T$ action on $X$ is not connected. Let $F=F^{1}+\cdots+F^{s}$ be the connected components of the fixed point set, $s \geqq 2$. We say that a subtorus $L$ connects $F^{1}$ and $F^{2}$ if they lie in the same component of $X^{L}$. We assume that $\operatorname{dim} H^{*}(X)$ is finite.

3. THEOREM. Let $N \subset H_{T}^{*}(X)$ be the ideal generated by odd degree and $R$ torsion elements. Assume that $H_{T}^{*}(X) / N$ is generated by $k$ elements as an $R$ algebra. Then for every maximal subtorus $K$ connecting $F^{1}$ and $F^{2}$, $\operatorname{rank} K \geqq \operatorname{rank} T-k$.

AMS (MOS) subject classifications (1970). Primary 55C20, 57E10, 57E25; Secondary $55 \mathrm{C} 35$. 
4. RemarK. This generalizes a result of Hsiang [3] that $F$ is connected whenever $H_{T}^{*}(X)$ is generated as an $R$ algebra by odd degree and $R$ torsion elements.

The following proposition is a technical result related to a theorem of Golber [2].

5. Proposition. Assume that $\operatorname{dim} H^{*}(X)=\operatorname{dim} H^{*}(F)<\infty$. Let $S=$ $\left\{x \in X \mid \operatorname{rank} T_{x} \geqq \operatorname{rank} T-1\right\}$. Then the homomorphism $H_{T}^{*}(X, F) \rightarrow$ $H_{T}^{*}(S, F)$ is injective.

We use the notation $X \sim Y$ to indicate that there is an isomorphism of rational cohomology rings $H^{*}(X)=H^{*}(Y)$. When $X \sim S^{k_{1}} \times \cdots \times S^{k_{n}}$ where the $k_{i}$ are odd integers, we define $e(X)$ to be the second symmetric polynomial $\sum_{i<j}\left(k_{i}+1\right)\left(k_{j}+1\right)$. If $\operatorname{dim} H^{*}(X)=\operatorname{dim} H^{*}(F)$, we know that $X^{L} \sim S^{d_{1}} \times \cdots \times S^{d_{n}}$ where the $d_{i}$ are odd integers, for every subtorus $L$ of $T$ [3]. Hence $e\left(X^{L}\right)$ is defined. Further we define $g(X)=$ $e(X)-e(F)-\sum_{L}\left[e\left(X^{L}\right)-e(F)\right]$ where $L$ ranges over the corank 1 subtori. For each subtorus $H$ of corank 2, we define $g\left(X^{H}\right)$ by using the induced $T / H$ action on $X^{H}$.

6. Proposition. $g(X)=\sum_{H} g\left(X^{H}\right)$ where $H$ ranges over the corank 2 subtori.

7. REMARK. Golber [2] has proved that $g(X)=\sum g\left(X^{H}\right)$ when $X \sim S^{k_{1}} \times S^{k_{2}}$ where the $k_{i}$ are odd, and $F=\varnothing$.

When $X$ is a compact rational cohomology manifold and $F=$ $F^{1}+\cdots+F^{s}$ are the components of the fixed point set, let $f_{i}$ be a generator of the top dimensional cohomology group of $F^{i}$. After including $f_{i} \in H^{*}\left(F^{i}\right) \subset H^{*}(F) \subset H_{T}^{*}(F)$, we can define the ideal $I_{f_{i}}$. The following result was conjectured by Hsiang. It is a kind of splitting principle or Schur lemma for torus actions.

8. THEOREM. The ideal $I_{f_{i}}$ is principal with a generator of degree $\operatorname{dim} X-\operatorname{dim} F^{i}$. This generator splits as a product of linear factors in $R$ corresponding to the subtori belonging to $f_{i}$.

Here $n=\operatorname{dim} X$ means that $H^{n}(X)$ is the top dimensional nonzero cohomology group of $X$. We do an explicit computation of $I_{f_{i}}$ when $X \sim$ quaternionic projective $n$ space [1].

9. Remark. Theorem 8 holds for torus actions on Poincaré duality spaces. It also holds for actions of $p$-tori on Poincaré duality spaces over $Z_{p}$. The Borel formula (see [3]) also holds for such actions [5].

Theorem 8 yields the following result of Hsiang and $\mathrm{Su}$ [4].

10. THEOREM. When $X$ is a compact rational cohomology manifold and $X \sim Q P^{n}$, quaternionic projective $n$ space, and a torus of rank $\geqq 2$ acts 
effectively on $X$, the fixed point set has at most one component $\sim Q P^{k}$ with $k \geqq 1$.

The results announced here also hold for actions of $p$-tori using $Z_{p}$ cohomology.

\section{REFERENCES}

1. T. Chang and T. Skjelbred, The topological Schur lemma and related results, Ann. of Math. (to appear).

2. D. Golber, Torus actions on a product of two odd spheres, Topology 10 (1971), 313-326. MR 44 \#1024.

3. Wu-yi Hsiang, On some fundamental theorems in cohomology theory of topological transformation groups, Taita J. Math. 2 (1970), 66-87.

4. Wu-yi Hsiang and J. C. Su, On the geometric weights system of topological actions on cohomology quaternionic projective spaces, Taipei-Berkeley-Amherst (mimeographed).

5. T. Skjelbred, Thesis, University of California, Berkeley, Calif., 1972.

Department of Mathematics, University of Kansas, LaWrence, Kansas 66044

Department of Mathematics, University of CAlifornia, Berkeley, California 94720

Current address (Tor Skjelbred): The Institute for Advanced Study, Princeton, New Jersey 08540 